\title{
¿Qué técnicas de comunicación oral valoran más los profesores $y$ alumnos en la clase de inglés?
}

José Luis OrTEGa MARTíN

DANIEL MADRID

Facultad de Ciencias de la Educación (Universidad de Granada)

Recibido: 15 marzo 2008 / Aceptado: 5 marzo 2009

ISSN: $1697-7467$

RESUMEN: Este artículo presenta un estudio sobre la percepción de profesores y alumnos de diferentes niveles educativos sobre la importancia que tienen, en la clase de inglés, determinadas técnicas de comprensión y expresión oral e informa en qué grado las consideran útiles para una buena enseñanza y aprendizaje de la lengua extranjera. Para ello, se ha empleado una muestra de 400 alumnos de varios niveles académicos y 59 profesores y se ha aplicado un cuestionario con 12 ítems correspondientes a doce técnicas de compresión oral y 11 ítems relacionados con once técnicas de expresión oral donde el alumnado y profesorado ha expresado su opinión usando una escala de tipo Likert con puntuaciones de 1 (= nada importante) a 5 (= muy importante). Los resultados nos dan a conocer las actividades de mayor y menor relevancia para profesores y alumnos de los distintos niveles académicos y demuestran la repercusión social que tiene la Selectividad y su poderosa influencia en las opiniones de los informantes.

Palabras clave: técnicas de comprensión y expresión oral en la clase de inglés, inglés como lengua extranjera.

What oral communication techniques do teachers and students attach a high value?

ABSTRACT: This paper presents a research study on students' and teachers' perceptions, of several academic levels, in relation to several oral comprehension and oral production classroom techniques and provides information as to what extent these activities are considered useful for an efficient teaching and learning of English as a Foreign Language. To this purpose, a questionnaire including 12 oral comprehension and 11 oral expression variables has been administered to a sample of 400 students and 59 teachers of several academic levels, who have expressed their perceptions by using a Likert scale from 1 (= very little importance) to 5 (= very important). The results obtained illustrate the most important oral techniques for students and teachers in a classroom setting and the washback effect of the Spanish university entrance examination on the informants' perceptions.

Key words: oral comprehension and oral expression techniques, English as a foreign language, university entrance examination, wahsback effect. 


\section{LAS DESTREZAS DE COMUNICACIÓN ORAL}

Para que los alumnos desarrollen su competencia oral con la lengua extrajera tienen que practicar cierta variedad de técnicas y estrategias de comprensión y expresión oral. Unas veces se limitan a escuchar y actúan de receptores de mensajes; otras hablan, producen mensajes y actúan como emisores y, en ocasiones, hay una alternancia entre el emisor y el receptor y se producen situaciones interactivas. Cuando el aprendiz participa en procesos de comunicación escrita tiene que poner en funcionamiento su capacidad lectora y su habilidad con la escritura; lo que se denomina en el ámbito anglohablante "literacy".

La división del lenguaje en cuatro destrezas -comprensión oral, expresión oral, lectura y escritura- toma fuerza con el método audiolingual en los años sesenta y ha tenido una gran aceptación en años posteriores en el plano didáctico y pedagógico para la enseñanza de las lenguas extranjeras.

Por otra parte, cuando el aprendizaje de la LE se produce en contextos formales, en el aula, se emplean cierta variedad de técnicas que combinan diversas estrategias. Estas estrategias constituyen el medio que el usuario de la lengua pone en funcionamiento para activar destrezas y procedimientos variados y cumplir con las demandas de las situaciones comunicativas.

\subsection{La comprensión oral}

Como indicamos en Corpas y Madrid (2009), sabemos que la adquisición de la LE incluye procesos de comunicación oral y escrita pero las situaciones de comprensión oral son más frecuentes que las demás: se estima que el 40-50\% del tiempo nos lo pasamos oyendo, el $25-30 \%$ hablando, el 10-16\% leyendo y el 10\% restante escribiendo. Por tanto, la importancia del desarrollo de la comprensión oral es evidente, pues el objetivo general de aprender una lengua extranjera no es otro que el de poder comunicarse, y, para ello, el hablante deberá tener la capacidad de recibir información, procesarla y entenderla para poder producir una respuesta coherente.

A veces se clasifica la comprensión oral y la lectura como destrezas pasivas a diferencia de la producción oral y escrita, que se consideran activas. Pero sería erróneo confundir la aparente actividad física con la inactividad mental. Para McLaren (1996: 214-217), escuchar una lengua extranjera no es una actividad pasiva en absoluto, sino extremadamente activa dado que:

- lo que se escucha realmente no son palabras, sino sonidos y silencios, que tendremos que procesar para extraer el significado del mismo en forma de palabras, frases y oraciones;

- además, el oyente tendrá que anticiparse a lo que va a venir y recordar lo que ya ha escuchado, por lo que si escucha una oración del tipo: "Él lo habría comprado si...", habrá de esperar que a continuación llegue una oración condicional, aunque puede ocurrir que no, y que el pronombre se refiera a una persona de la que se ha hablado anteriormente.

Además, creemos que hay una serie de factores que contribuyen a que esta destreza se convierta en una tarea activa, como advierte McLaren (1996: 213-214): 
- Escuchar es como leer. Si escuchamos atentamente los sonidos uno por uno como si fuesen letras, podremos reconocer las palabras.

- Cuando estamos escuchando, lo importante es reconocer cada palabra que el hablante dice. Hay que preocuparse de entender las palabras, y el significado de la oración vendrá solo.

- Escuchar es, básicamente, un juego de averiguación. Debemos pensar en lo que parece que el hablante dice e intentar averiguar lo que quiere decir a partir de lo que creemos que quiere comunicar.

- La comprensión oral puede ser difícil, así que necesitamos toda la ayuda posible. Conviene escuchar atentamente los sonidos que emite el hablante $\mathrm{y}$, al mismo tiempo, usar nuestros conocimientos de la gramática y del vocabulario y nuestras mejores artes para averiguar lo que quiere decir.

Como hemos indicado en otros trabajos, creemos que escuchar es importante porque (Madrid y McLaren, 1995: 39):

- Proporciona información esencial para el proceso de la comunicación.

- Se necesita en la vida real.

- Es algo que se puede trabajar en grupo.

- Permite a los alumnos distinguir sonidos, acentuación y patrones de entonación fundamentales para la comunicación oral.

- Conecta con lo que el alumno hace a menudo: escuchar música, ver cadenas de televisión musicales, vídeos, etc.

- Una comprensión oral exitosa puede aumentar la motivación para el aprendizaje de las lenguas.

En contextos de aula, entre las destrezas que se ponen en marcha en los procesos de comprensión oral podríamos destacar las siguientes:

- La discriminación de sonidos en palabras sueltas o conectadas en un discurso.

- La discriminación de patrones de acentuación.

- El reconocimiento de la acentuación y de las variantes de ésta.

- El reconocimiento de aquellas palabras o grupos de palabras que indiquen el desarrollo de una idea, la explicación o clarificación, etc.

- La distinción de la idea principal y su discriminación de otras de menor importancia.

- La capacidad de recoger los puntos esenciales del texto para poder resumirlo.

- La capacidad de eliminar o no prestar atención a aquellos elementos que no son importantes para la comprensión del texto.

- La comprensión de lo que se quiere indicar con los distintos patrones de entonación, es decir, si el hablante presenta una actitud u otra.

- La información que no aparece de manera explícita en el texto.

- Las relaciones dentro de la oración y de las partes de un texto.

- El significado conceptual, en particular de cantidad, comparación, causa, resultado, etc.

Las opciones del profesorado a la hora de proponer actividades de comprensión oral son múltiples (véase Anderson y Lynch, 1988; McLaren, 1996; White, 1998; Tejada y Pérez Cañado, 2004; Harris y Hewitt, 2005). Se pueden ofrecer, entre otras: 
- Actividades de representación: el alumno puede oír el texto y gesticular lo que en él se dice, asegurándose así el profesor su comprensión.

- Actividades de entrenamiento auditivo: con estas actividades se entrena al alumno para que reconozca entonación, secuencias de sonidos, segmentos realmente significativos y morfemas y palabras que puedan causar confusión. (Rivers y Temperley, 1978: 98).

- Actividades en las que se aprenda a distinguir claramente el mensaje en situaciones que puedan crear confusión, especialmente con conectores, pronombres, preposiciones, modales, prefijos, morfemas, etc.

- Actividades en las que se discriminen sonidos (juegos como el bingo o ejercicios de escuchar y leer), el acento o la entonación.

- Diálogos: simples del tipo de los libro de texto, o más largos en los que el alumno no tiene que fijarse en cada una de las palabras que lo componen sino en el texto como un conjunto.

- La audición de narraciones, lo que es sin duda una de las actividades más populares y útiles pero que presenta, entre otras, dificultades como que el texto puede ser un largo monólogo en el que toda la información sea relevante con la dificultad de no poder identificar a los distintos personajes al haber un único narrador o que sea necesario un gran dominio del estilo directo e indirecto para su comprensión.

Dentro de esta amplia gama, en el cuestionario que hemos aplicado a profesores y alumnos (véase apéndice 1), intentamos conocer la importancia que le conceden a la realización de algunas de las actividades anteriores.

\subsection{La expresión oral}

Hablar una lengua lleva consigo entenderla oralmente, es decir, la producción y la comprensión oral interactúan entre sí en la comunicación oral. Varias teorías han enfatizado la importancia del "input" recibido y de la interacción en la adquisición de la segunda lengua. Una de las más conocidas ha sido la hipótesis del input comprensible de Krashen (1985), que mantiene que la lengua se adquiere mejor cuando el aprendiz entiende el mensaje que recibe. Por otro lado, Ellis (1984: 95) mantiene que la interacción contribuye al desarrollo de esta destreza porque es el medio por el cual el aprendiz es capaz de romper el código. Para Klein (1986) estas dos teorías se complementan ya que tanto el input comprensible como las oportunidades de comunicación son condiciones necesarias para que se dé el aprendizaje.

Tejada y Nieto (1996: 242-3) señalan los siguientes rasgos que hay que tener en cuenta como característicos del lenguaje empleado en los procesos de producción oral y que lo diferencian del escrito:

- Enunciados cortos, normalmente oraciones o frases incompletas así como expresiones ya hechas.

- Predominio de la voz activa sobre la pasiva.

- Estructura de lo comentado: una vez que el tema se cita y lo entiende el oyente, se añade algún comentario. 
- Se hace referencia al entorno.

- Se usa un vocabulario muy general con un bajo grado de explicitud.

- Se admite la repetición de enunciados o trozos de éstos.

- Se usan pausas frecuentes, interrupciones, marcadores y rellenos para clarificar, reelaborar o comprobar la comprensión, todo en una atmósfera de espontaneidad.

Para Rivers y Temperley (1978: 4), todo el proceso de aprendizaje de la comunicación oral y escrita ocurre en dos fases:

\section{Fase Cognitiva:}

- Conocimiento de componentes de la comunicación: reglas, categorías, funciones, .

- Interiorizar las reglas referidas con aquellas categorías y funciones.

\section{Fase Productiva:}

- Práctica de secuencias de sonidos (que pueden incluir "palabras").

- Práctica en la formulación de comunicaciones (incluye oraciones, mensajes cortos y textos más extensos).

En los procesos de comunicación oral que tienen lugar en el aula, Tejada y Nieto (1996: 246-248) hablan de una primera fase pre-comunicativa y una segunda fase en la que el alumno realice actividades encaminadas a conseguir fluidez. Proponen las siguientes actividades:

Fase 1.

- Práctica oral: se buscarán ejercicios en los que la respuesta se active si se ha entendido la información dada y con la intención de trabajar sobre todo el orden de las palabras, los morfemas o los fonemas del enunciado.

- Memorización de diálogos: útil, sobre todo, para facilitar marcos discursivos además de proporcionar mejoras a nivel sintáctico. Los diálogos deberán ser abiertos para que el alumno pueda tomar la iniciativa e introducir sus propios comentarios.

- Juegos orales para instrucciones y preguntas: en estas actividades los alumnos dan instrucciones o hacen preguntas para obtener una respuesta ya sea física o verbal. Se pueden usar materiales como tarjetas, fotos, "realia" o textos.

- Dictado de símbolos: se han de rellenar los huecos de una fotografía o de un diagrama con dibujos fáciles siguiendo las instrucciones de un hablante. Se podrá comprobar el éxito del proceso viendo que efectivamente el dibujo obtenido sea fruto de la descripción.

- Grabaciones: se propone la grabación de las conversaciones de los alumnos, anuncios, entrevistas o llamadas telefónicas como si se tratase de una emisora de radio.

Fase 2.

- Discusiones: se facilitan distintos temas para la discusión, como podrían ser las noticias, las experiencias propias o cualquier evento.

- Juegos con dibujos y fotos: se persigue provocar la conversación por medio de ayuda visual usando, por ejemplo, dos fotografías para encontrar similitudes y/o diferencias, etc. 
- Cuentos y discursos: hacer que los alumnos den pequeños discursos o cuenten cuentos.

- Simulaciones y representaciones: se da a los alumnos diferentes papeles para que resuelvan diferentes situaciones.

En el cuestionario que hemos empleado para estudiar las percepciones de profesores y alumnos sobre la importancia de las técnicas de producción oral en el aula hemos incluido sólo once técnicas, a modo de variables (véase apéndice 1), que suelen ser bastante usuales en contextos de aula (véase también Bygate, 1987).

\section{Metodología de InVestigación}

\subsection{Objetivos}

En nuestro trabajo nos planteamos conocer la opinión de profesores y alumnos sobre la importancia que tienen en la clase de idioma determinadas técnicas de comprensión y expresión oral y estas dos destrezas globalmente consideradas. También tratamos de estudiar si la valoración de alumnos y profesores va en función de variables descriptivas tales como género, edad, tipo de centro, etc. y daremos a conocer las actividades de comunicación oral unidireccional y bidireccional preferidas por profesores y alumnos.

\subsection{Muestra}

La muestra ha sido seleccionada según un tipo de muestreo no probabilístico casual en el que el criterio de selección de los individuos depende de la posibilidad de acceder a ellos. El total ha sido de 455 sujetos distribuidos del siguiente modo:

\begin{tabular}{|l|l|c|c|}
\hline \multicolumn{4}{|c|}{ ALUMNOS $=402$} \\
\hline \multirow{4}{*}{ Género } & Categoría & $N$ & $\%$ \\
\hline \multirow{5}{*}{ Centro } & Hombres & 261 & 64,9 \\
\cline { 2 - 4 } & Mujeres & 141 & 35,1 \\
\cline { 2 - 4 } & Público & 112 & 27,9 \\
\cline { 2 - 4 } & Privado & 239 & 59,5 \\
\cline { 2 - 4 } & Universidad & 51 & 12,7 \\
\hline \multirow{5}{*}{ Rendimiento } & $6^{\text {o }}$ Primaria & 24 & 6 \\
\cline { 2 - 4 } & 1er Ciclo ESO & 58 & 14 \\
\cline { 2 - 4 } & $2^{\circ}$ Ciclo ESO & 135 & 33,6 \\
\cline { 2 - 4 } & Bachillerato & 134 & 33,2 \\
\cline { 2 - 4 } & Universidad & 51 & 12,7 \\
\cline { 2 - 4 } & Alto & 143 & 36,9 \\
\cline { 2 - 4 } & Medio & 152 & 39,2 \\
\hline
\end{tabular}




\section{PROFESORES:}

Los datos referidos al profesorado se reflejan en la tabla siguiente:

\begin{tabular}{|c|c|c|c|}
\hline \multicolumn{4}{|c|}{ PROFESORES $=53$} \\
\hline & Categoría & $\mathrm{N}$ & $\%$ \\
\hline \multirow[t]{2}{*}{ Género } & Hombres & 23 & 43,4 \\
\hline & Mujeres & 30 & 56,6 \\
\hline \multirow{4}{*}{ Centro } & Público & 20 & 37,7 \\
\hline & Privado & 7 & 13,2 \\
\hline & En prácticas & 18 & 34 \\
\hline & Universidad & 8 & 15,1 \\
\hline \multirow{6}{*}{$\begin{array}{l}\text { Nivel } \\
\text { educativo }\end{array}$} & $6^{\circ}$ Primaria & 2 & 3,8 \\
\hline & 1er Ciclo ESO & 3 & 5,7 \\
\hline & $2^{\circ}$ Ciclo ESO & 10 & 18,9 \\
\hline & Bachillerato & 12 & 22 \\
\hline & Universidad & 8 & 15,1 \\
\hline & En prácticas & 18 & 34,5 \\
\hline \multirow{2}{*}{$\begin{array}{l}\text { Experiencia } \\
\text { docente }\end{array}$} & Menos de 5 años & 41 & 77,4 \\
\hline & 5 o más años & 12 & 22,6 \\
\hline \multirow[t]{3}{*}{ Edad } & Menores de 30 años & 38 & 71,7 \\
\hline & De 31 a 45 años & 12 & 22,6 \\
\hline & Mayores de 45 años & 3 & 5,7 \\
\hline \multirow{3}{*}{$\begin{array}{l}\text { Tendencia } \\
\text { política }\end{array}$} & Izquierda & 15 & 53,6 \\
\hline & Centro & 6 & 21,4 \\
\hline & Derecha & 7 & 25 \\
\hline
\end{tabular}

\section{Diseño experimental}

Para la elaboración del cuestionario hemos seleccionado los procedimientos y técnicas descritas en el mismo, adaptándolos para una mayor comprensión de los informantes. A los profesores, por su parte, se les hizo entrega del cuestionario para que procediesen a su cumplimentación sin poner una limitación de tiempo.

En todos los casos se han respetado las normas deontológicas o éticas exigidas en la investigación humana. También se ha respetado el derecho a que la información sea confidencial y que el instrumento y la situación de la investigación sean pensadas para recoger una conducta esperada en condiciones normales.

Por todo lo expuesto, el diseño que utilizamos es un procedimiento de investigación cuantitativa no experimental de método descriptivo y correlacional. El método descriptivo de estudios de encuesta se basa en la formulación de preguntas a personas que poseen la información y la comunican a través de un cuestionario. El método correlacional pretende describir las relaciones que existen entre las variables que intervienen en un fenómeno. En los procedimientos cualitativos, utilizamos el método fenomenológico para centrarnos en cómo las personas comprenden los significados de lo que sucede. Esta metodología parece adecuarse para el objetivo de nuestra investigación. 


\subsection{Definición de variables}

En el cuestionario del apéndice 1 indicamos las variables controladas para realizar el estudio en función de los objetivos marcados. Aparecen variables descriptivas para los alumnos (curso que estudia o edad, tipo de centro, género, rendimiento) y para los profesores (género, tipo de centro en el que imparte, años de docencia y edad, nivel en el que imparte). También aparecen 23 variables que resumen, en nuestra opinión, las técnicas de comprensión y expresión oral más usuales en la clase de LE. Estas variables se han valorado según una escala Lickert, según el criterio siguiente: 1: nada importante; 2: poco importante; 3: aceptable 4: importante; 5: muy importante.

Las clases de variables utilizadas han sido las siguientes: cualitativas de tipo nominal, cualitativas de tipo ordinal y cuantitativas discretas.

\section{Variables descriptivas del alumno:}

Curso que estudia o edad: variable cualitativa nominal. Se han asignado los siguientes valores: 1: primaria; 2: ESO; 3: Bachillerato; 4: Universidad.

Tipo de centro: variable cualitativa nominal. Se han asignado los siguientes valores: 1: titularidad privada; 2: titularidad pública; 3: Universidad.

Género: variable cualitativa nominal y dicotómica. Se han asignado los siguientes valores: 1: hombre; 2: mujer.

Rendimiento: variable cualitativa ordinal. Se han asignado los siguientes valores: 1: bajo (0-4); 2: normal (5-6); 3: bueno (7-10).

\section{Variables descriptivas del profesor:}

Tipo de centro en el que imparte: variable cualitativa nominal. Se han asignado los siguientes valores: 1: titularidad privada; 2: titularidad pública; 3: universidad.

Nivel en el que imparte: variable cualitativa nominal. Se han asignado los siguientes valores: 1: Primaria; 2: ESO1; 3: ESO2; 4: Bachillerato; 5: Universidad; 6: Profesorado de Prácticas.

Distinguimos entre ESO1 y ESO2 porque hay profesorado que, siendo maestros de primaria, imparten docencia en este ciclo.

\subsection{Tratamiento estadístico}

El procedimiento estadístico ha sido:

Descriptivo. Se ha realizado el estudio de las variables definidas. En él aparecen los parámetros típicos de tendencia central (media, moda, etc.) y de desviación (desviación típica y varianza) así como diagramas de barras y tantos por cientos válidos y acumulados.

Validación. Se han calculado parámetros de fiabilidad (Alfa de Cronbach) y validez factorial para dotar de fiabilidad-validez a los cuestionarios usados en el estudio, ya que han sido elaboradas por el propio autor.

Inferencias. Las pruebas utilizadas se han aplicado en función de las características de 
las variables. Así, se han utilizado pruebas no paramétricas como: Mann Whitney, KruskalWallis, Friedman, Wilcoxon.

\section{Resultados}

\subsection{Fiabilidad del Cuestionario}

Hemos calculado la fiabilidad de los cuestionarios que se han utilizado en nuestro estudio, para ver hasta qué punto las preguntas medían el mismo objetivo. Se ha utilizado el "Alfa de Crombach". Los resultados fueron los siguientes:

\begin{tabular}{|l|c|c|}
\hline & Profesores & Alumnos \\
\hline Ítems de comprensión oral & 0.82 & 0.83 \\
\hline Ítems de expresión oral & 0.84 & 0,80 \\
\hline
\end{tabular}

\subsection{Resultados relacionados con la Comprensión Oral}

Los profesores de Secundaria coinciden en señalar que el desarrollo de la comprensión oral es esencial y se le ha de prestar mucha atención por su importancia en el proceso de comunicación, pero consideran que es muy difícil de evaluar.

Para la mayoría del profesorado en prácticas, el dominio de esta destreza es muy importante, pero aclaran que no se debe considerar más importante que otras destrezas, sino que ha de formar parte de un todo, pues las cuatro destrezas son igualmente importantes, lo ideal es una integración de todas ellas. Hay quien aún mantiene, quizás por la influencia de la Selectividad, que la comprensión oral, especialmente en Bachillerato, es menos importante que la expresión escrita o la lectura.

El profesorado de universidad piensa que es esencial y, aunque es un componente muy importante, se suele descuidar dando prioridad a lo escrito, lo cual es un error. Se recomienda que se potencie desde los primeros años de enseñanza.

El alumnado de Secundaria y Bachillerato prefiere que el input oral proceda del profesor porque a veces las grabaciones no se oyen bien. Como en el examen de Selectividad no se pide comprensión oral, algunos alumnos no le conceden la importancia que merece.

El alumnado de universidad considera que la comprensión oral es de gran utilidad, pero también insiste en que las cuatro destrezas deben trabajarse por igual porque las conversaciones, ya sean diálogos, monólogos o debates en público, fomentan la comunicación en otra lengua y "sirven para desinhibirnos y hacernos entender". También reconocen su importancia para el posterior desarrollo de la expresión oral.

Los datos cuantitativos que nos proporciona el profesorado han arrojado los siguientes resultados (Ortega, 2003: 568-569): 


\begin{tabular}{|c|c|c|c|c|c|c|}
\hline \multirow[b]{2}{*}{ VARIABLES (COMPRENSIÓN ORAL) } & & \multicolumn{5}{|c|}{ Nivel académico del profesorado } \\
\hline & & Prim & $\mathrm{Sec}$ & Bach & Univ & CAP \\
\hline \multirow[b]{2}{*}{ 1. Escuchar una audición e identificar el tema general } & M & 4.67 & 4.31 & 4.17 & 4.29 & 3.92 \\
\hline & Sig & \multicolumn{5}{|l|}{.418} \\
\hline \multirow{2}{*}{$\begin{array}{l}\text { 2. Escuchar una audición e identificar datos } \\
\text { específicos }\end{array}$} & M & 3.67 & 4.15 & 3.92 & 4.14 & 3.92 \\
\hline & $\operatorname{sig}$ & \multicolumn{5}{|l|}{.636} \\
\hline \multirow{2}{*}{$\begin{array}{l}\text { 3. Comprender oralmente la actitud del hablante, el } \\
\text { carácter y tono de sus intervenciones, etc. }\end{array}$} & M & 4.00 & 3.92 & 3.83 & 3.71 & 3.43 \\
\hline & sig & \multicolumn{5}{|l|}{.296} \\
\hline \multirow{2}{*}{$\begin{array}{l}\text { 4. Realizar audiciones de diálogos, textos, películas, } \\
\text { etc en la LE. }\end{array}$} & M & 4.80 & 3.85 & 4.00 & 3.86 & 4.17 \\
\hline & sig & \multicolumn{5}{|l|}{.170} \\
\hline \multirow{2}{*}{$\begin{array}{l}\text { 5. Comprensión oral de significados conceptuales } \\
\text { como cantidad, comparaciones, tiempo, ubicación, } \\
\text { etc. }\end{array}$} & M & 4.33 & 3.83 & 3.58 & 3.86 & 3.29 \\
\hline & sig & \multicolumn{5}{|l|}{.177} \\
\hline \multirow{2}{*}{$\begin{array}{l}\text { 6. Comprensión oral de la relación entre las partes de } \\
\text { un texto }\end{array}$} & M & 4.00 & 3.92 & 3.33 & 3.14 & 3.42 \\
\hline & sig & \multicolumn{5}{|l|}{.141} \\
\hline \multirow{2}{*}{$\begin{array}{l}\text { 7. Identificar la información principal de un texto y } \\
\text { distinguirla de elementos secundarios }\end{array}$} & $\mathrm{M}$ & 4.80 & 4.38 & 3.83 & 3.43 & 4.17 \\
\hline & sig & \multicolumn{5}{|l|}{.055} \\
\hline \multirow{2}{*}{$\begin{array}{l}\text { 8. Informar antes de oír el texto de situación, temática } \\
\text { y vocabulario específico que pudiera causar confusión }\end{array}$} & M & 4.67 & 4.08 & 4.00 & 3.86 & 3.71 \\
\hline & sig & \multicolumn{5}{|l|}{.601} \\
\hline \multirow{2}{*}{$\begin{array}{l}\text { 9. Oír textos adaptados al nivel del alumnado para que } \\
\text { la mayor parte del "input" sea comprensible }\end{array}$} & M & 5.00 & 4.31 & 4.42 & 4.14 & 4.33 \\
\hline & sig & \multicolumn{5}{|l|}{.503} \\
\hline \multirow{2}{*}{$\begin{array}{l}\text { 10. Insistir en que no hay que entender cada una de las } \\
\text { palabras de un texto para comprenderlo oralmente }\end{array}$} & M & 4.67 & 4.77 & 4.58 & 4.71 & 4.63 \\
\hline & sig & \multicolumn{5}{|l|}{.910} \\
\hline \multirow{2}{*}{$\begin{array}{l}\text { 11. Realizar actividades de comprensión y } \\
\text { dramatización de lo que se está escuchando }\end{array}$} & M & 4.67 & 4.08 & 4.08 & 3.86 & 3.96 \\
\hline & sig & \multicolumn{5}{|l|}{.647} \\
\hline \multirow[b]{2}{*}{ 12. Escuchar secuencias de narraciones o historias } & M & 4.33 & 4.00 & 3.42 & 3.57 & 3.63 \\
\hline & sig & \multicolumn{5}{|l|}{.317} \\
\hline
\end{tabular}

Puede observarse que el profesorado expresa una valoración muy alta respecto a las técnicas de comprensión oral, siendo los profesores de Primaria los que más valoran estas técnicas. No existen diferencias significativas entre los profesores de los distintos niveles excepto en relación a la variable 7 (Identificar la información principal de un texto y distinguirla de elementos secundarios) que se valora mucho más en Primaria que entre el profesorado universitario.

Los resultados del alumnado en relación a las técnicas de comprensión oral son los siguientes: 


\begin{tabular}{|c|c|c|c|c|c|c|c|}
\hline \multirow{2}{*}{\multicolumn{2}{|c|}{ VARIABLES (COMPRENSIÓN ORAL) }} & \multicolumn{3}{|c|}{ Tipo de centro } & \multicolumn{3}{|c|}{ Rendimiento } \\
\hline & & \multirow{2}{*}{$\begin{array}{ll}\text { Priv } \\
3.58 \\
\end{array}$} & \multirow{2}{*}{$\begin{array}{l}\text { Públ } \\
3.34 \\
\end{array}$} & \multirow{2}{*}{$\frac{\text { Univ }}{4.10}$} & \multirow{2}{*}{$\frac{\text { Bajo }}{3.37}$} & \multirow{2}{*}{$\frac{\text { Medio }}{3.59}$} & \multirow{2}{*}{$\frac{\text { Alto }}{3.65}$} \\
\hline \multirow{2}{*}{$\begin{array}{l}\text { 1. Escuchar una audición e identificar el tema } \\
\text { general }\end{array}$} & $\mathrm{M}$ & & & & & & \\
\hline & sig & \multicolumn{3}{|l|}{.000} & \multicolumn{3}{|l|}{.121} \\
\hline \multirow{2}{*}{$\begin{array}{l}\text { 2. Escuchar una audición e identificar datos } \\
\text { específicos }\end{array}$} & M & 3.48 & 3.19 & 3.88 & 3.23 & 3.39 & 3.64 \\
\hline & sig & \multicolumn{3}{|l|}{.000} & \multicolumn{3}{|l|}{.006} \\
\hline \multirow{2}{*}{$\begin{array}{l}\text { 3. Comprender oralmente la actitud del hablante, } \\
\text { el carácter y tono de sus intervenciones, }\end{array}$} & $\mathrm{M}$ & 3.43 & 3.19 & 3.82 & 3.32 & 3.31 & 3.54 \\
\hline & sig & \multicolumn{3}{|l|}{.001} & \multicolumn{3}{|l|}{.037} \\
\hline \multirow{2}{*}{$\begin{array}{l}\text { 4. Realizar audiciones de diálogos, textos, } \\
\text { películas, etc en la LE. }\end{array}$} & M & 3.83 & 3.49 & 4.02 & 3.71 & 3.66 & 3.91 \\
\hline & sig & \multicolumn{3}{|l|}{.009} & \multicolumn{3}{|l|}{.081} \\
\hline \multirow{2}{*}{$\begin{array}{l}\text { 5. Comprensión oral de significados } \\
\text { conceptuales como cantidad, comparaciones, } \\
\text { tiempo, ubicación, etc. }\end{array}$} & $\mathrm{M}$ & 3.29 & 3.04 & 3.75 & 3.18 & 3.18 & 3.42 \\
\hline & sig & \multicolumn{3}{|l|}{.000} & \multicolumn{3}{|l|}{.058} \\
\hline \multirow{2}{*}{$\begin{array}{l}\text { 6. Comprensión oral de la relación entre las } \\
\text { partes de un texto }\end{array}$} & M & 3.27 & 3.16 & 3.82 & 3.21 & 3.27 & 3.37 \\
\hline & sig & \multicolumn{3}{|l|}{.000} & \multicolumn{3}{|l|}{.435} \\
\hline \multirow{2}{*}{$\begin{array}{l}\text { 7. Identificar la información principal de un texto } \\
\text { y distinguirla de elementos secundarios }\end{array}$} & M & 3.36 & 3.13 & 3.92 & 3.15 & 3.24 & 3.61 \\
\hline & sig & \multicolumn{3}{|l|}{.000} & \multicolumn{3}{|l|}{.001} \\
\hline \multirow{2}{*}{$\begin{array}{l}\text { 8. Informar antes de oír el texto de situación, } \\
\text { temática y vocabulario específico que pudiera } \\
\text { causar confusión }\end{array}$} & M & 3.31 & 3.23 & 3.73 & 3.15 & 3.30 & 3.42 \\
\hline & sig & \multicolumn{3}{|l|}{.012} & .201 & & \\
\hline 9. Oír textos adaptados al nivel del alumnado & M & 3.67 & 3.48 & 3.94 & 3.50 & 3.62 & 3.76 \\
\hline 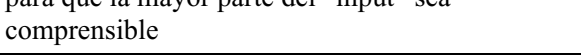 & sig & .064 & & & .188 & & \\
\hline 10. Insistir en que no hay que entender cada una & M & 3.54 & 3.49 & 4.24 & 3.43 & 3.52 & 3.83 \\
\hline oralmente & sig & .000 & & & .011 & & \\
\hline 11. Realizar actividades de comprensión y & $\mathrm{M}$ & 3.40 & 3.23 & 3.78 & 3.30 & 3.39 & 3.45 \\
\hline dramatización de lo que se está escuchando & sig & .002 & & & .441 & & \\
\hline & M & 3.46 & 3.16 & 3.71 & 3.27 & 3.31 & 3.56 \\
\hline historias & sig & .005 & & & .039 & & \\
\hline
\end{tabular}

Puede observarse que el alumnado valora mucho menos que el profesorado las técnicas de comprensión oral, quizás porque cuesta trabajo realizarlas y porque los medios técnicos disponibles no son adecuados en todos los casos y las audiciones no se escuchan adecuadamente. También nos llama la atención las diferencias tan fuertes entre las valoraciones del alumnado de los diferentes centros, ya que son estadísticamente significativas en relación a casi todas las variables. Son los alumnos del nivel universitario los que más valoran todas estas técnicas de comprensión oral en contraste con los de los centros públicos, que les conceden menos importancia. También nos llama la atención el hecho de que sean los alumnos de alto rendimiento académico los que más valoran estas actividades en contraste con los 
de bajo rendimiento, que les conceden puntuaciones más bajas. Esas diferencias son especialmente significativas en relación a la variable 7. (Identificar la información principal de un texto y distinguirla de elementos secundarios). Como hemos indicado anteriormente, esta diferencia puede deberse al alto nivel de dificultad que suelen tener estas actividades y a la poca atención que se les presta en clase por la escasa infraestructura técnica de que dispone el profesorado para llevarlas a cabo (equipos de sonido de poca calidad, aulas con ruido, etc.)

Agrupados los alumnos por género y nivel de estudios, los resultados fueron los siguientes:

\begin{tabular}{|c|c|c|c|c|c|c|c|}
\hline & & \multicolumn{2}{|c|}{ Género } & \multicolumn{4}{|c|}{ Nivel de estudios } \\
\hline \multicolumn{2}{|l|}{ VARIABLES (COMPRENSIÓN ORAL) } & M & $\mathrm{F}$ & Prim & $\mathrm{Sec}$ & Bach & Univ \\
\hline \multirow{2}{*}{$\begin{array}{l}\text { 1. Escuchar una audición e identificar el tema } \\
\text { general }\end{array}$} & M & 3.48 & 3.76 & 3.48 & 3.54 & 3.45 & 4.00 \\
\hline & sig & \multicolumn{2}{|l|}{.012} & \multicolumn{4}{|l|}{.005} \\
\hline \multirow{2}{*}{$\begin{array}{l}\text { 2. Escuchar una audición e identificar datos } \\
\text { específicos }\end{array}$} & M & 3.38 & 3.58 & 3.50 & 3.41 & 3.33 & 3.75 \\
\hline & sig & \multicolumn{2}{|l|}{.059} & \multicolumn{4}{|l|}{.073} \\
\hline \multirow{2}{*}{$\begin{array}{l}\text { 3. Comprender oralmente la actitud del hablante, } \\
\text { el carácter y tono de sus intervenciones, etc. }\end{array}$} & M & 3.31 & 3.62 & 3.48 & 3.37 & 3.31 & 3.67 \\
\hline & sig & \multicolumn{2}{|l|}{.001} & \multicolumn{4}{|l|}{.082} \\
\hline \multirow{2}{*}{$\begin{array}{l}\text { 4. Realizar audiciones de diálogos, textos, } \\
\text { películas, etc. en la LE. }\end{array}$} & M & 3.67 & 3.92 & 3.78 & 3.73 & 3.75 & 3.94 \\
\hline & sig & \multicolumn{2}{|l|}{.021} & \multicolumn{4}{|l|}{.492} \\
\hline \multirow{2}{*}{$\begin{array}{l}\text { 5. Comprensión oral de significados conceptuales } \\
\text { como cantidad, comparaciones, tiempo, ubicación, } \\
\text { etc. }\end{array}$} & $\mathrm{M}$ & 3.17 & 3.46 & 3.39 & 3.17 & 3.23 & 3.69 \\
\hline & sig & \multicolumn{2}{|l|}{.004} & \multicolumn{4}{|l|}{.002} \\
\hline \multirow{2}{*}{$\begin{array}{l}\text { 6. Comprensión oral de la relación entre las partes } \\
\text { de un texto }\end{array}$} & $\mathrm{M}$ & 3.24 & 3.43 & 3.48 & 3.18 & 3.28 & 3.73 \\
\hline & sig & \multicolumn{2}{|l|}{.058} & \multicolumn{4}{|l|}{.001} \\
\hline \multirow{2}{*}{$\begin{array}{l}\text { 7. Identificar la información principal de un texto } \\
\text { y distinguirla de elementos secundarios }\end{array}$} & $\mathrm{M}$ & 3.21 & 3.65 & 3.29 & 3.27 & 3.41 & 3.73 \\
\hline & sig & \multicolumn{2}{|l|}{.000} & \multicolumn{4}{|l|}{.036} \\
\hline \multirow{2}{*}{$\begin{array}{l}\text { 8. Informar antes de oír el texto de situación, } \\
\text { temática y vocabulario específico que pudiera } \\
\text { causar confusión }\end{array}$} & $\mathrm{M}$ & 3.22 & 3.55 & 3.36 & 3.22 & 3.39 & 3.75 \\
\hline & sig & \multicolumn{2}{|l|}{.001} & \multicolumn{4}{|l|}{.007} \\
\hline \multirow{2}{*}{$\begin{array}{l}\text { 9. Oír textos adaptados al nivel del alumnado para } \\
\text { que la mayor parte del "input" sea comprensible }\end{array}$} & $\mathrm{M}$ & 3.56 & 3.82 & 3.67 & 3.51 & 3.81 & 4.13 \\
\hline & sig & \multicolumn{2}{|l|}{.026} & \multicolumn{4}{|l|}{.002} \\
\hline \multirow{2}{*}{$\begin{array}{l}\text { 10. Insistir en que no hay que entender cada una } \\
\text { de las palabras de un texto para comprenderlo } \\
\text { oralmente }\end{array}$} & M & 3.50 & 3.83 & 3.41 & 3.51 & 3.72 & 4.19 \\
\hline & sig & \multicolumn{2}{|l|}{.004} & .000 & & & \\
\hline 11. Realizar actividades de comprensión y & M & 3.32 & 3.54 & 3.61 & 3.31 & 3.28 & 3.75 \\
\hline dramatización de lo que se está escuchando & sig & .027 & & .005 & & & \\
\hline & M & 3.34 & 3.53 & 3.48 & 3.36 & 3.31 & 3.69 \\
\hline 12. Escuchar secuencias de narraciones o historias & sig & .080 & & .110 & & & \\
\hline
\end{tabular}


También hay diferencias considerables en las valoraciones del alumnado agrupado por género y nivel de estudios. Desde el punto de vista del género, estas diferencias son significativas en relación a cinco variables, a favor de las chicas, que valoran más las actividades de comprensión oral que los chicos.

En la agrupación del alumnado por nivel de estudios, también se observan diferencias significativas en relación a seis de las doce variables. En este caso son los alumnos de Primaria los que valoran menos estas técnicas de trabajo en contraste con los universitarios que son los que les conceden una puntuación más alta.

Si comparamos los resultados entre profesores y alumnos, observamos que aunque unos y otros coinciden al señalar la importancia del desarrollo de esta destreza, la valoración de los profesores es mayor que la de los alumnos:

\begin{tabular}{|c|c|c|c|c|c|c|}
\hline \multicolumn{7}{|c|}{ COMPRENSIÓN ORAL } \\
\hline \multicolumn{4}{|c|}{ ALUMNOS } & \multicolumn{3}{|c|}{ PROFESORES } \\
\hline Variables & $\mathrm{N}$ & Media & Desv.Tip. & $\mathrm{N}$ & Media & Desv.Tip. \\
\hline 1 & 400 & 3,58 & 1,030 & 59 & 4,14 & ,776 \\
\hline 2 & 400 & 3,45 & 1,000 & 59 & 3,98 & 629 \\
\hline 3 & 400 & 3,42 & 957 & 58 & 3,69 &, 883 \\
\hline 4 & 399 & 3,76 & 1,041 & 59 & 4,07 & ,785 \\
\hline 5 & 398 & 3,28 & ,970 & 58 & 3,59 &, 817 \\
\hline 6 & 400 & 3,31 & ,951 & 58 & 3,50 &, 822 \\
\hline 7 & 399 & 3,37 & ,999 & 58 & 4,09 & ,823 \\
\hline 8 & 399 & 3,34 & ,999 & 59 & 3,92 & 1,005 \\
\hline 9 & 400 & 3,65 & 1,041 & 59 & 4,36 & ,783 \\
\hline 10 & 399 & 3,62 & 1,082 & 59 & 4,66 &, 576 \\
\hline 11 & 400 & 3,40 & ,981 & 59 & 4,03 & ,787 \\
\hline 12 & 400 & 3,41 & 1,017 & 59 & 3,69 & 915 \\
\hline
\end{tabular}

Debido a que el examen de Selectividad para ingreso en la universidad hasta la fecha no ha incluido prueba de comprensión oral son los alumnos y profesores de Bachillerato los que, aun reconociendo la importancia de esta destreza, creen que es poco útil para cumplir con sus objetivos académicos.

De una forma gráfica, la diferencia entre las puntuaciones de profesores y alumnos se ilustra en la figura siguiente: 


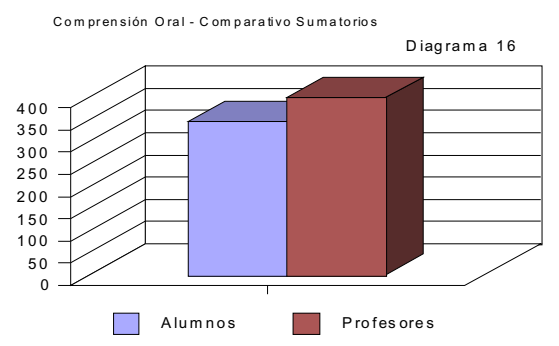

\subsection{Resultados relacionados con la Producción Oral}

El profesorado de Secundaria considera que las actividades de expresión oral son muy importantes aunque también reconoce que se valora más lo escrito que lo oral. Los profesores de prácticas (CAP), por su parte, siguen manteniendo, en su mayoría, que lo importante es la combinación de destrezas. Mantienen que este tipo de actividades "deben comenzar a ponerse en práctica en los primeros niveles de la enseñanza". El profesorado de universidad también coincide en la especial importancia de esta destreza, aunque lo conveniente es que se combinen y se integren varias destrezas.

Las opiniones de los alumnos son variadas y han resaltado varios puntos. Consideran que hay que dedicar tiempo a este tipo de actividades ya que son muy importantes para comunicarse en la lengua extranjera y viajar al extranjero. Creen que es importante practicar con el profesor y con otros compañeros. Advierten que hay que trabajar esta destreza pero no se deben abandonar las otras y hay quienes piensan que el profesor tiene que obligar a los alumnos a que hablen sólo en inglés en las clases. En el sector universitario siguen reconociendo la gran utilidad de la producción oral e insisten en que las cuatro destrezas deben trabajarse por igual. Dado que estas actividades son fundamentales para dominar la lengua extranjera, habría que erradicar el miedo a hablar en clase y procurar que no sientan miedo de hacer el ridículo cuando hablan inglés en clase.

En relación a las actividades de producción oral, los resultados obtenidos con el profesorado fueron los siguientes (véase Ortega, 2003: 572-575): 


\begin{tabular}{|c|c|c|c|c|c|c|}
\hline \multirow[b]{2}{*}{ VARIABLES DE EXPRESIÓN ORAL } & & \multicolumn{5}{|c|}{ Nivel que imparte } \\
\hline & & Prim & $\mathrm{Sec}$ & Bach & Univ & CAP \\
\hline \multirow{2}{*}{$\begin{array}{l}\text { 13. Actividades de producción oral guiadas y } \\
\text { controladas }\end{array}$} & M & 4.50 & 4.08 & 3.92 & 4.29 & 3.96 \\
\hline & sig & \multicolumn{5}{|l|}{.774} \\
\hline \multirow[b]{2}{*}{ 14.Actividades de producción libres } & M & 4.00 & 3.77 & 3.58 & 3.29 & 3.50 \\
\hline & sig & \multicolumn{5}{|l|}{.912} \\
\hline \multirow{2}{*}{$\begin{array}{l}\text { 15. Responder a preguntas formuladas por el profesor } \\
\text { o por el compañero }\end{array}$} & M & 4.33 & 4.08 & 4.08 & 4.43 & 4.12 \\
\hline & sig & \multicolumn{5}{|l|}{.774} \\
\hline \multirow[b]{2}{*}{ 16. Realizar entrevistas adaptadas en dificultad } & M & 4.33 & 4.15 & 3.92 & 3.43 & 3.88 \\
\hline & sig & \multicolumn{5}{|l|}{.498} \\
\hline \multirow{2}{*}{$\begin{array}{l}\text { 17. Simular situaciones en que el alumno desempeñe } \\
\text { roles variados y representarlas oralmente }\end{array}$} & M & 4.67 & 4.38 & 4.17 & 3.57 & 4.00 \\
\hline & sig & \multicolumn{5}{|l|}{.191} \\
\hline \multirow{2}{*}{$\begin{array}{l}\text { 18.Uso de muletillas (fillers) del tipo "o sea, quiero } \\
\text { decir, sabes" en la expresión oral }\end{array}$} & $\mathrm{M}$ & 3.67 & 3.08 & 3.17 & 3.29 & 3.21 \\
\hline & sig & \multicolumn{5}{|l|}{.888} \\
\hline \multirow{2}{*}{$\begin{array}{l}\text { 19.Favorecer la comunicación oral con diálogos } \\
\text { abiertos }\end{array}$} & $\mathrm{M}$ & 3.67 & 3.85 & 3.83 & 3.57 & 3.88 \\
\hline & sig & \multicolumn{5}{|l|}{.953} \\
\hline \multirow{2}{*}{$\begin{array}{l}\text { 20.Usar la mímica, los gestos y el movimiento físico } \\
\text { para la comunicación oral }\end{array}$} & $\mathrm{M}$ & 4.33 & 3.69 & 3.75 & 3.71 & 3.46 \\
\hline & sig & \multicolumn{5}{|l|}{.546} \\
\hline \multirow{2}{*}{$\begin{array}{l}\text { 21. Hacer preguntas sobre láminas, dibujos o } \\
\text { fotografías para practicar la producción oral }\end{array}$} & $M$ & 4.67 & 3.92 & 3.92 & 3.71 & 4.00 \\
\hline & sig & \multicolumn{5}{|l|}{.428} \\
\hline \multirow{2}{*}{$\begin{array}{l}\text { 22. Presentar oralmente argumentos a favor y en } \\
\text { contra de un tema }\end{array}$} & $\mathrm{M}$ & 4.50 & 3.69 & 4.17 & 3.29 & 3.75 \\
\hline & sig & \multicolumn{5}{|l|}{.359} \\
\hline \multirow{2}{*}{$\begin{array}{l}\text { 23. Inventar cuentos o historias a partir de elementos } \\
\text { claves dados }\end{array}$} & $M$ & 3.33 & 3.85 & 3.67 & 3.71 & 3.58 \\
\hline & sig & \multicolumn{5}{|l|}{.844} \\
\hline
\end{tabular}

Puede observarse que el profesorado de todos los niveles educativos valora mucho las técnicas de expresión oral, en especial el de Primaria. Las puntuaciones son bastantes homogéneas y no se han obtenido diferencias significativas.

En relación a las puntuaciones del alumnado, agrupado por tipo de centro y rendimiento académico, hemos obtenido los siguientes resultados: 


\begin{tabular}{|c|c|c|c|c|c|c|c|}
\hline \multirow{2}{*}{\multicolumn{2}{|c|}{ VARIABLES }} & \multicolumn{3}{|c|}{ Tipo de centro } & \multicolumn{3}{|c|}{ Rendimiento } \\
\hline & & \multirow{2}{*}{$\frac{\text { Priv }}{3.49}$} & \multirow{2}{*}{$\frac{\text { Públ }}{3.28}$} & \multirow{2}{*}{$\begin{array}{l}\text { Univ } \\
3.94\end{array}$} & \multirow{2}{*}{$\frac{\text { Bajo }}{3.29}$} & \multirow{2}{*}{$\frac{\text { Medio }}{3.43}$} & \multirow{2}{*}{$\frac{\text { Alto }}{3.65}$} \\
\hline \multirow{2}{*}{$\begin{array}{l}\text { 13. Actividades de producción oral guiadas y } \\
\text { controladas }\end{array}$} & M & & & & & & \\
\hline & sig & \multicolumn{3}{|l|}{.000} & \multicolumn{3}{|l|}{.029} \\
\hline \multirow[b]{2}{*}{ 14.Actividades de producción libres } & $\mathrm{M}$ & 3.11 & 2.87 & 3.37 & 3.03 & 2.97 & 3.18 \\
\hline & sig & \multicolumn{3}{|l|}{.019} & \multicolumn{3}{|l|}{.172} \\
\hline \multirow{2}{*}{$\begin{array}{l}\text { 15. Responder a preguntas formuladas por el } \\
\text { profesor o por el compañero }\end{array}$} & M & 3.55 & 3.37 & 3.86 & 3.49 & 3.46 & 3.63 \\
\hline & sig & \multicolumn{3}{|l|}{.011} & \multicolumn{3}{|l|}{.434} \\
\hline \multirow[b]{2}{*}{ 16. Realizar entrevistas adaptadas en dificultad } & M & 3.48 & 3.38 & 4.04 & 3.42 & 3.51 & 3.56 \\
\hline & $\operatorname{sig}$ & \multicolumn{3}{|l|}{.000} & \multicolumn{3}{|l|}{.595} \\
\hline \multirow{2}{*}{$\begin{array}{l}\text { 17. Simular situaciones en que el alumno } \\
\text { desempeñe roles variados y representarlas } \\
\text { oralmente }\end{array}$} & M & 3.47 & 3.32 & 3.86 & 3.35 & 3.49 & 3.54 \\
\hline & $\operatorname{sig}$ & \multicolumn{3}{|l|}{.016} & \multicolumn{3}{|l|}{.198} \\
\hline \multirow{2}{*}{$\begin{array}{l}\text { 18.Uso de muletillas (fillers) del tipo "o sea, } \\
\text { quiero decir, sabes" en la expresión oral }\end{array}$} & M & 3.14 & 2.89 & 3.43 & 2.91 & 3.13 & 3.19 \\
\hline & sig & \multicolumn{3}{|l|}{.016} & \multicolumn{3}{|l|}{.197} \\
\hline \multirow{2}{*}{$\begin{array}{l}\text { 19.Favorecer la comunicación oral con } \\
\text { diálogos abiertos }\end{array}$} & M & 3.58 & 3.12 & 3.88 & 3.24 & 3.45 & 3.68 \\
\hline & sig & \multicolumn{3}{|l|}{.000} & \multicolumn{3}{|l|}{.007} \\
\hline \multirow{2}{*}{$\begin{array}{l}\text { 20.Usar la mímica, los gestos y el movimiento } \\
\text { físico para la comunicación oral }\end{array}$} & M & 2.92 & 2.97 & 3.41 & 2.90 & 2.96 & 3.06 \\
\hline & sig & \multicolumn{3}{|l|}{.037} & \multicolumn{3}{|l|}{.437} \\
\hline 21. Hacer preguntas sobre láminas, dibujos o & M & 3.22 & 3.16 & 3.75 & 3.11 & 3.22 & 3.41 \\
\hline fotografías para practicar la producción oral & sig & .003 & & & .146 & & \\
\hline 22 Presentar oralmente aroumentos a favor v & M & 3.22 & 3.23 & 3.82 & 2.98 & 3.34 & 3.43 \\
\hline en contra de un tema & sig & .001 & & & .006 & & \\
\hline 23 Inventar cuentos 0 historias a nartir de & M & 3.27 & 2.88 & 3.73 & 3.16 & 3.16 & 3.27 \\
\hline elementos claves dados & sig & .000 & & & .616 & & \\
\hline
\end{tabular}

Resulta interesante observar que no hay diferencias significativas entre el alumnado agrupado por rendimiento académico, sin embargo sí que hay cuando se agrupa por tipo de centro. Lo mismo que ocurría con las técnicas de comprensión oral, hay diferencias significativas entre el alumnado de los centros privados y públicos a la hora de valorar estas actividades. De nuevo se pone de manifiesto que el alumnado de los centros privados valora más las técnicas de producción oral que el de los centros públicos.

Los resultados del alumnado agrupado según el factor género y nivel de estudios fueron los siguientes: 


\begin{tabular}{|c|c|c|c|c|c|c|c|}
\hline \multirow{2}{*}{\multicolumn{2}{|c|}{ VARIABLES }} & \multicolumn{2}{|c|}{ Género } & \multicolumn{4}{|c|}{ Nivel de estudios } \\
\hline & & & \multirow{2}{*}{\begin{tabular}{|l|}
$\mathrm{F}$ \\
3.70
\end{tabular}} & \multirow{2}{*}{$\frac{\text { Prim }}{3.61}$} & \multirow{2}{*}{$\frac{\mathrm{Sec}}{3.36}$} & \multirow{2}{*}{$\frac{\text { Bach }}{3.51}$} & \multirow{2}{*}{$\frac{\text { Univ }}{3.92}$} \\
\hline \multirow{2}{*}{$\begin{array}{l}\text { 13. Actividades de producción oral guiadas y } \\
\text { controladas }\end{array}$} & M & & & & & & \\
\hline & sig & \multicolumn{2}{|l|}{.002} & \multicolumn{4}{|l|}{.005} \\
\hline \multirow[b]{2}{*}{ 14.Actividades de producción libres } & M & 3.07 & 3.09 & 3.17 & 3.01 & 3.08 & 3.21 \\
\hline & sig & \multicolumn{2}{|l|}{.818} & \multicolumn{4}{|l|}{.564} \\
\hline \multirow{2}{*}{$\begin{array}{l}\text { 15. Responder a preguntas formuladas por el } \\
\text { profesor o por el compañero }\end{array}$} & M & 3.46 & 3.67 & 3.76 & 3.43 & 3.57 & 3.73 \\
\hline & sig & \multicolumn{2}{|l|}{.024} & \multicolumn{4}{|l|}{.069} \\
\hline \multirow[b]{2}{*}{ 16. Realizar entrevistas adaptadas en dificultad } & M & 3.41 & 3.72 & 3.54 & 3.42 & 3.60 & 3.88 \\
\hline & sig & \multicolumn{2}{|l|}{.010} & \multicolumn{4}{|l|}{.038} \\
\hline \multirow{2}{*}{$\begin{array}{l}\text { 17. Simular situaciones en que el alumno } \\
\text { desempeñe roles variados y representarlas } \\
\text { oralmente }\end{array}$} & M & 3.42 & 3.60 & 3.65 & 3.40 & 3.49 & 3.58 \\
\hline & sig & \multicolumn{2}{|l|}{.091} & \multicolumn{4}{|l|}{.387} \\
\hline \multirow{2}{*}{$\begin{array}{l}\text { 18.Uso de muletillas (fillers) del tipo "o sea, } \\
\text { quiero decir, sabes" en la expresión oral }\end{array}$} & M & 3.11 & 3.12 & 3.20 & 3.05 & 3.13 & 3.25 \\
\hline & sig & \multicolumn{2}{|l|}{.945} & \multicolumn{4}{|l|}{.760} \\
\hline \multirow{2}{*}{$\begin{array}{l}\text { 19.Favorecer la comunicación oral con diálogos } \\
\text { abiertos }\end{array}$} & M & 3.39 & 3.67 & 3.41 & 3.42 & 3.51 & 3.83 \\
\hline & sig & \multicolumn{2}{|l|}{.011} & \multicolumn{4}{|l|}{.117} \\
\hline \multirow{2}{*}{$\begin{array}{l}\text { 20.Usar la mímica, los gestos y el movimiento } \\
\text { físico para la comunicación oral }\end{array}$} & M & 2.88 & 3.21 & 3.24 & 2.92 & 2.83 & 3.35 \\
\hline & sig & \multicolumn{2}{|l|}{.005} & \multicolumn{4}{|l|}{.028} \\
\hline & M & 3.20 & 3.40 & 3.24 & 3.16 & 3.35 & 3.69 \\
\hline fotografías para practicar la producción oral & sig & .048 & & .017 & & & \\
\hline 22. Presentar oralmente argumentos a favor y & M & 3.19 & 3.49 & 3.52 & 3.16 & 3.33 & 3.63 \\
\hline en contra de un tema & sig & .006 & & .013 & & & \\
\hline & M & 3.19 & 3.27 & 3.17 & 3.14 & 3.35 & 3.37 \\
\hline elementos claves dados & $\operatorname{sig}$ & .445 & & .262 & & & \\
\hline
\end{tabular}

Puede observarse que las puntaciones del alumnado según el factor género y nivel de estudios son mucho más homogéneas que las obtenidas con las técnicas de comprensión oral. Es decir, hay mayor grado de coincidencia a la hora de valorar estas actividades y consiguientemente menos diferencias significativas. Las únicas diferencias significativas que hemos obtenido se relacionan con la variable 1 (Actividades de producción oral guiadas y controladas) y 8 (Usar la mímica, los gestos y el movimiento físico para la comunicación oral), en ambos casos a favor de las chicas, que las consideran más importantes que los chicos.

Si comparamos las valoraciones de profesores y alumnos observamos que tanto unos como otros coinciden en que la expresión oral es una destreza muy importante y hay que dedicarle más tiempo. La queja es común en cuanto a que debiera comenzar a trabajarse con más intensidad desde los primeros años de aprendizaje de la lengua extranjera. 
Tanto profesores como alumnos consideran útiles la simulación de situaciones en las que el alumno desempeña roles variados y los ha de representar oralmente, aunque más los primeros que los segundos (profesores Med. = 4,10; alumnos Med. = 3,48). También los profesores valoran más que los alumnos el uso de la mímica, los gestos y el movimiento físico como elementos paralingüísticos que facilitan la comprensión (profesores Med. = 3,64; alumno Med. $=3,00)$.

\begin{tabular}{|c|c|c|c|c|c|c|}
\hline \multicolumn{7}{|c|}{ EXPRESIÓN ORAL } \\
\hline \multicolumn{4}{|c|}{ ALUMNOS } & \multicolumn{3}{|c|}{ PROFESORES } \\
\hline VARIABLES & $\mathrm{N}$ & Media & Desv,. Tip. & $\mathrm{N}$ & Media & Desv Típ \\
\hline 13 & 400 & 3,49 & 1,006 & 58 & 4,03 & ,816 \\
\hline 14 & 400 & 3,08 & 1,101 & 59 & 3,58 & 1,004 \\
\hline 15 & 398 & 3,54 & ,953 & 59 & 4,15 & 690 \\
\hline 16 & 400 & 3,52 & 1,040 & 59 & 3,92 & ,794 \\
\hline 17 & 400 & 3,48 & 1,071 & 59 & 4,10 & ,781 \\
\hline 18 & 398 & 3,11 & 1,139 & 59 & 3,20 & 1,013 \\
\hline 19 & 398 & 3,49 & 1,062 & 59 & 3,81 & ,798 \\
\hline 20 & 400 & 3,00 & 1,150 & 59 & 3,64 & ,905 \\
\hline 21 & 400 & 3,27 & 1,089 & 59 & 3,97 &, 718 \\
\hline 22 & 400 & 3,30 & 1,087 & 58 & 3,79 & ,969 \\
\hline 23 & 399 & 3,22 & 1,130 & 59 & 3,66 & 822 \\
\hline
\end{tabular}

La gráfica que ilustra la comparación de las valoraciones de profesores y alumnos es muy similar a la de la comprensión oral como puede apreciarse a continuación:

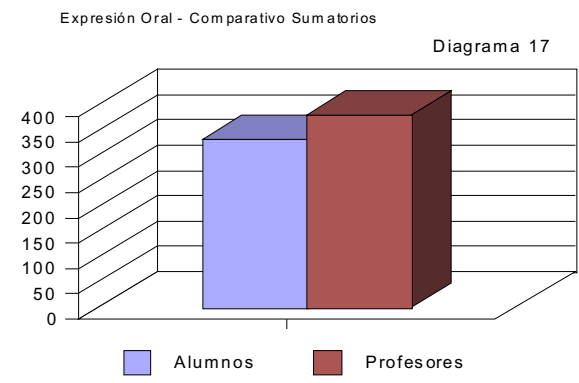




\section{Conclusiones}

\subsection{Comprensión Oral}

Las actividades de comprensión oral que más valoran profesores y alumnos, de entre las evaluadas, son las siguientes:

- Realizar audiciones de diálogos, textos, películas, etc. en la LE

- Oír textos adaptados al nivel del alumnado para que la mayor parte del input sea comprensible

- Escuchar una audición e identificar el tema general y los datos específicos

Sin embargo las dos actividades menos valoradas son la comprensión oral de la relación entre las partes de un texto y la comprensión oral de significados conceptuales tales como: cantidad, comparaciones y tiempo, etc.

De las cuatro destrezas, la comprensión oral es la más valorada (profesores: $M=3,99$; alumnos $\mathrm{M}=3,46$ ). Las valoraciones más altas, entre el alumnado, proceden de los alumnos de mayor rendimiento, lo que nos lleva a pensar que este tipo de alumnos se desenvuelve mejor con los ejercicios orales y valora más el desarrollo de su competencia comunicativa.

La valoración de todas y cada una de las variables estudiadas para esta destreza es mayor en el caso de las alumnas que en los alumnos, aunque las diferencias no son significativas.

Son los alumnos universitarios los que valoran más esta destreza, seguidos por los de Primaria y ESO.

La baja valoración que los alumnos de Bachillerato otorgan a las actividades de comprensión oral puede deberse a que no se exige en el examen de Selectividad.

\subsection{Producción Oral}

Las actividades de producción oral que más se valoran son las siguientes:

- Responder a preguntas formuladas por el profesor o por el compañero

- Realizar entrevistas adaptadas en dificultad

- Actividades de producción oral guiadas y controladas

- Favorecer la comunicación oral con diálogos abiertos

- Simular situaciones en que el alumno desempeñe roles variados y representarlas oral

Las menos valoradas han sido las actividades de producción libre (quizás porque les resultan difíciles de llevar a cabo) y el uso de la mímica, los gestos y el movimiento físico para la comunicación oral

Es evidente que las actividades que no son útiles para la Selectividad se valoran menos, pues no cabe duda de que las entrevistas adaptadas en dificultad son esenciales para el desarrollo de la comunicación oral del alumnado, pero como no hay entrevistas en la LE en dicho examen, estas actividades pasan a un segundo plano. Uno de los acontecimientos más importante para los alumnos y profesores de Bachillerato es el examen de la Selectividad y, cuando ése es el objetivo prioritario, profesores y alumnos se olvidan de lo oral para dedicar su tiempo al desarrollo de la escritura y la lectura. 
También sucede que la postura más cómoda del profesor es la de seguir un libro de texto en donde se suelen explotar las unidades por medio de actividades que implican la lectura o audición de un texto de introducción a la unidad, contestar a preguntas de comprensión sobre el mismo, explicación gramatical de los elementos nuevos y ejercicios gramaticales y de vocabulario para consolidar el lenguaje de la unidad, etc. Aunque en todos los textos aparecen actividades de producción oral, éstas suelen ser difíciles de llevar a cabo dada la poca homogeneidad de los grupos y el elevado número de alumnos.

\section{REFERENCIAS}

Anderson, A. and Lynch, T. (1988). Listening. Oxford: Oxford University Press

Bygate, M. (1987): Speaking. Oxford: Oxford University Press

Corpas M. D. y Madrid, D. (2009). "Desarrollo de la comprensión oral en inglés como LE al término de la Educación Secundaria Obligatoria española”. Porta Linguarum, $\mathrm{n}^{\circ} 11$, pp.129-145.

Ellis, R. (1984). Classroom Second Language Development. A Study of Classroom interaction and Language Acquisition. Oxford: Pergamon Press.

Harris, T y Hewitt, E. (2005). "Listening Comprensión", en McLaren, N. , Madrid, D. y Bueno, A. (eds.): TEFL in Secondary Education. Granada: Editorial Universidad de Granada, pp. 281-320.

Klein, W. (1986). Second Language Acquisition. Cambridge: Cambridge University Press.

Krashen, S. (1985). The Input Hypothesis: Issues and Implications. Torrante: Laredo P. Co., CA.

Madrid, D. y McLaren, N. (1995). Didactic Procedures for TEFL. Valladolid: La Calesa.

Madrid, D. y McLaren, N. (2004). TEFL in Primary Education. Granada: Editorial Universidad de Granada.

McLaren, N. (1996). “Listening Comprensión”, en McLaren, N. y D. Madrid (eds) (1996), pp. 209-328.

McLaren, N. y Madrid, D. (eds) (1996): A Handbook for TEFL. Alcoy: Marfil.

McLaren, N. , Madrid, D. y Bueno, A. (eds.) (2005). TEFL in Secondary Education. Granada: Editorial Universidad de Granada.

Ortega, J.L. (2003). Percepciones de profesores sobre la importancia de los principios y técnicas que se aplican en el aula. Granada: Grupo Editorial Universitario.

Rivers, W. M. y Temperley, M. S. (1978). A Practical Guide to the Teaching of English as A Second language. New York: Oxford University Press.

Tejada, G. y Nieto, J. (1996). “Oral Communication”, en McLaren, N. y D. Madrid (eds) (1996), pp. 209-328.

Tejada, G. y Pérez Cañado, M. L. (2004). “Oral Communication and Interaction”, en Madrid, D. y McLaren, N. (eds.), pp. 178-218.

White, G. (1998). Listening. Oxford: Oxford University Press. 


\section{APÉNDICE 1}

\section{Cuestionario del Alumnado y Profesorado}

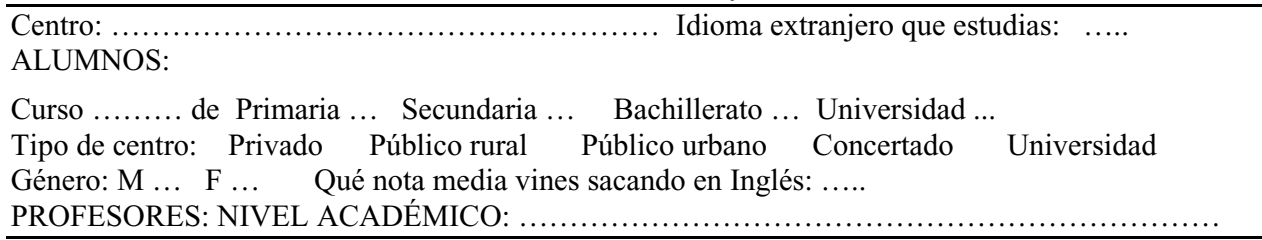

Lee con atención las actividades y técnicas de enseñana y aprendizaje siguientes e indica en qué grado los consideras importantes y útiles para una buena enseñanza y aprendizaje de la lengua extranjera. Puntúa cada aspecto de 1 a 5 aplicando la escala siguiente.

$$
1=\text { Nada importante } 2 \text { =Poco importante } 3 \text { =Aceptable } 4 \text { =importante } 5=\text { Muy importante }
$$

\section{Técnicas de Comprensión oral}

1. (.....) Escuchar una audición e identificar el tema general.

2. (.....) Escuchar una audición e identificar datos específicos.

3. (.....) Comprender oralmente la actitud del hablante, el carácter y tono de sus intervenciones, etc.

4. (.....) Realizar audiciones de diálogos, textos, películas, etc. en la LE.

5. (.....) Comprensión oral de significados conceptuales como cantidad, comparaciones, tiempo, ubicación, causa, resultado, propósito, etc.

6. (.....) Comprensión oral de la relación entre las partes de un texto.

7. (.....) Identificar la información principal de un texto y distinguirla de elementos secundarios.

8. (.....) Informar antes de oír el texto de la situación y la temática del mismo y del vocabulario específico que pudiera causar problemas de comprensión (pre-listening)

9. (.....)Oír textos adaptados al nivel del alumnado para que la mayor parte del "input" sea comprensible.

10. (.....) Insistir en que no hay que entender cada una de las palabras de un texto para comprenderlo oralmente.

11. (.....) Realizar actividades de comprensión y dramatización de lo que se está escuchando.

12. (.....) Escuchar secuencias de narraciones o historias.

\section{Técnicas de Expresión oral}

13. (.....) Actividades de producción oral guiadas y controladas por el profesor.

14. (.....) Actividades de producción libres en las que nadie controle lo que escribimos o decimos.

15. (.....) Responder a preguntas formuladas por el profesor o por el compañero.

16. (.....) Realizar entrevistas con compañeros o con el profesor adaptadas en dificultad.

17. (.....) Simular situaciones y representarlas oralmente.

18. (.....) Uso de muletillas (fillers) del tipo "you know what I mean”, “OK?”, etc. en la expresión oral.

19. (.....) Favorecer la comunicación oral con diálogos abiertos (en los que puedes añadir lo que quieras).

20. (.....) Usar la mímica, los gestos y el movimiento físico para la comunicación oral.

21. (.....) Que el profesor nos haga presentar oralmente argumentos a favor y en contra de un tema.

22. (.....) Contestar a preguntas sobre láminas, dibujos o fotografías para practicar la producción oral.

23. (.....) Inventar cuentos o historias a partir de elementos claves dados. 


\section{ASPECTOS CUALITATIVOS}

Utilidad e importancia de las actividades de comprensión oral y del desarrollo de esta destreza en el proceso de Enseñanza/aprendizaje de la lengua extranjera.

Utilidad e importancia de las actividades de expresión oral y del desarrollo de esta destreza en el proceso de enseñanza/ aprendizaje de la lengua extranjera.

Utilidad e importancia de las actividades en las que se integran dos o más destrezas en el proceso de enseñanza/aprendizaje de la lengua extranjera. 